\title{
Sick Sinus Syndrome Induced by Giant Coronary Artery Aneurysm Rupture in a Patient with Constrictive Pericarditis
}

\begin{abstract}
Keywords: Coronary artery a neurysm; Constric tive peric arditis; Sick sinus syndrome

Abstract

Multiple coronary artery a neurysms (CAA) with a giant CAA are rare. Patient with a giant CAA is at risk of thrombosis, a neurysm rupture and even sudden cardiac death. We reported a case of a 78-year-old woman having a giant CAA $(43 \mathrm{~mm} \times 37 \mathrm{~mm})$ of proximal left anterior descending artery as well as multiple small CAA of both left and right coronary arteries. The spontaneous rupture of the giant CAA combined with previously undiagnosed constric tive peric arditis leaded to severe dyspnea, edema of lower extremities, and syncope. Emergency electrocardiogram (ECG) showed remarkable sinus bradycardia, sinus a rest and a trioventric ular junc tional $\mathrm{mythm}$, ind ic ating sick sinus syndrome. Temporary cardiac pacemaker therapy was performed urgently to avoid sudden cardiac death. Multiple non-inva sive imaging tests revea led constric tive peric ard itis possibly caused by tuberculosis, and giant CAA with the possibility of rupture. Then peric ardiotomy and resection of giant aneurysm were performed urgently. Postoperative course was uneventful. The patient became asymptomatic and was discharged in good health. To the best of our knowledge, this is the first case of spontaneous rupture of giant CAA in a patient with constric tive peric a rditis, with a rare complic ation of sick sinus synd rome.
\end{abstract}

\section{Case Report}

A 78-year-old woman was referred to our emergency department because of severe dyspnea, edema of lower extremities, and syncope twice in the last two weeks. Before admission, the patient had mild dyspnea on exertion and edema of lower extremities for two years. But the patient did not go to hospital for medical consultation. Two weeks ago, the patient began to experience exacerbations of these symptoms and had syncope twice, without symptoms of chest pain, fever and cough. Her medical history included old tuberculosis.

On admission emergency electrocardiogram revealed remarkable sinus bradycardia (heart rate 42 beat per minute), sinus arrest and atrioventricular junctional rhythm (Figure 1A), indicating sick sinus syndrome. Because of severe dyspnea and edema of lower extremities, we did not try atropine test to rule out high vagal tone as a possible cause of transient bradycardia and junctional escape. Temporary cardiac pacemaker therapy was performed urgently to avoid sudden cardiac death. The chest radiograph revealed mild cardiac enlargement. Transthoracic echocardiogram demonstrated pericardial effusion, pericardial thickening with calcification, and a large cystic mass adjacent to pulmonary artery. Cardiac computed tomography (CT) showed massive pericardial effusion, obvious pericardial thickening, and a large vascular mass adjacent to pulmonary artery (Figure 2A). Computed tomography angiography (CTA) demonstrated that there was one giant CAA $(43 \mathrm{~mm} \times 37 \mathrm{~mm})$

\section{Journal of}

\section{Cardiobiology}

Yang Liu',2\#, Ping Hua ${ }^{3 \#}$, Jie Chen², Huan-Ji Zhang $^{1,2}$, Wei-Feng Sun ${ }^{1}$, Rong Zou ${ }^{2}$, Hui Huang ${ }^{1,2 *}$, Jing-Feng Wang ${ }^{1,2 *}$

${ }^{1}$ Department of Cardiology, Sun Yat-sen Memorial Hospital, Sun Yat-sen University, Guangzhou, 510120 China

${ }^{2}$ Key Laboratory of Cardiac Electrophysiology and Arrhythmia, Guangdong Province, Guangzhou, 510120 China

${ }^{3}$ Department of Thoracic Surgery, Sun Yat-sen Memorial Hospital, Sun Yat-sen University, Guangzhou, 510120 China

"These authors equally contributed

*Address for Correspondence

Hui Huang, MD, PhD, Department of Cardiology, Sun Yat-sen Memorial Hospital of Sun Yat-sen University, 107 West Yanjiang Road, Guangzhou, China 510120, Tel: 0086-20-81332475; Fax: 0086-20-81332623; E-mail: huanghui765@hotmail.com

Jing-Feng Wang, MD, PhD, Department of Cardiology, Sun Yat-sen Memorial Hospital of Sun Yat-sen University, 107 West Yanjiang Road, Guangzhou, China 510120, Tel: 0086-20-81332475; Fax: 0086-2081332623; E-mail: drwjfsums@gmail.com

Copyright: $\odot 2014$ Liu Y, et al. This is an open access article distributed under the Creative Commons Attribution License, which permits unrestricted use, distribution, and reproduction in any medium, provided the original work is properly cited.

Submission: 29 January, 2014

Accepted: 19 February, 2014

Published: 24 February, 2014

Reviewed \& Approved by: Dr. Xiaojie Xie, Department of Cardiology, Second Affiliated Hospital, Zhejiang University School of Medicine, China

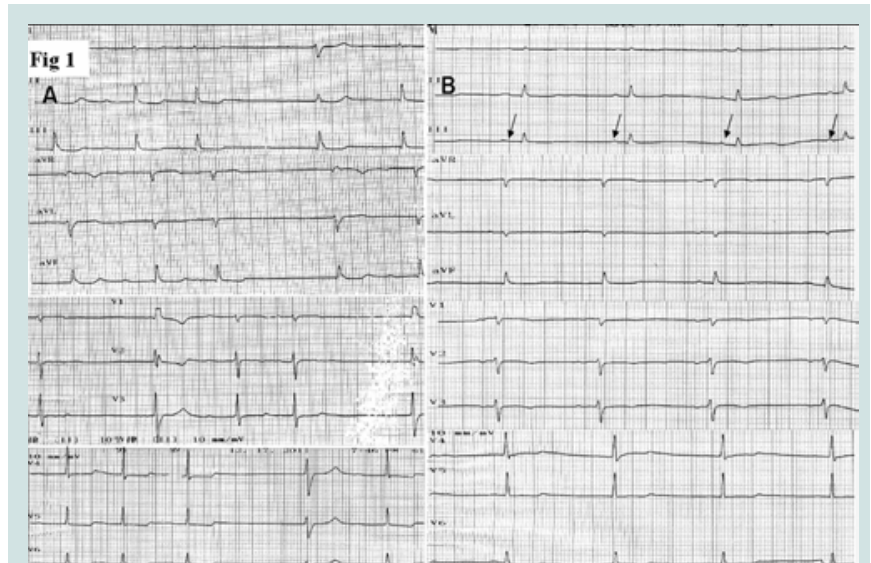

Figure 1: (A) sinus arrest and atrioventricular junctional rhythm before treatment; (B) normal rhythm after treatment.

of proximal left anterior descending artery with multiple small CAA of both left and right coronary arteries (Figure 2C). We considered the possibility of aneurysm rupture. Thus median sternotomy and pericardiotomy were performed urgently. The operation revealed significant pericardial thickening $(3-5 \mathrm{~mm})$, extensive pericardial adhesions, hemopericardium and aneurysm rupture. There was no obvious atherosclerotic lesions and thrombus in the giant aneurysm. Surgical exploration found the giant aneurysm having four malformed fed arteries from both left and right coronary arteries. Then the adhesions of pericardium were lysed, the fed arteries were ligated, and the giant aneurysm was resected (Figure 3). We did not consider bypass graft in this patient, because there were many small aneurysms 
around the giant one and the resection of aneurysm had no significant impact on blood supply. Postoperative histopathological findings demonstrated that pericardium was thickened with calcification and hyalold degeneration, and that aneurysmal wall showed significant fibrous thickening with hyalold degeneration, mucoid degeneration and chronic lymphoplasmacytic cell infiltration. Besides PPD test, other tests on tuberculosis including hydrothorax smear and mycobacterium tuberculosis culture were negative. Tests on thoracic cancer and autoimmune diseases were also negative. Postoperative course was uneventful. The patient had no recurrence of sinus dysfunction after operation and was discharged in good condition (Figure 1B, 2B and 2D).

\section{Discussion}

From the findings above, we came to the diagnosis of spontaneous rupture of giant CAA and constrictive pericarditis with a rare complication of sick sinus syndrome. Multiple CAA with one giant CAA are rare [1]. To the best of our knowledge, this is the first case of spontaneous rupture of giant coronary artery aneurysm in a patient with constrictive pericarditis. The causes of giant CAA include atherosclerosis, Kawasaki disease, Takayasu arteritis, and so on [2]. We did not determine the final cause of aneurysms in this case. Although atherosclerosis is the main cause of giant CAA [2], we found no obvious atherosclerotic lesions and the patient had no history of smoking, hypertension, diabetes, hyperlipidemia and coronary artery disease. As the patient had old tuberculosis and possibly tuberculous constrictive pericarditis, we cannot exclude Takayasu arteritis secondary to tuberculosis as the etiology. There are a few cases on CAA possibly caused by tuberculosis [3,4]. Multiple CAA are also found to be secondary to Kawasaki disease [5-7], but there was no clue on Kawasaki disease in our case. Giant CAA may also be congenital in origin [8]. In our case, no other contributory factors were found.
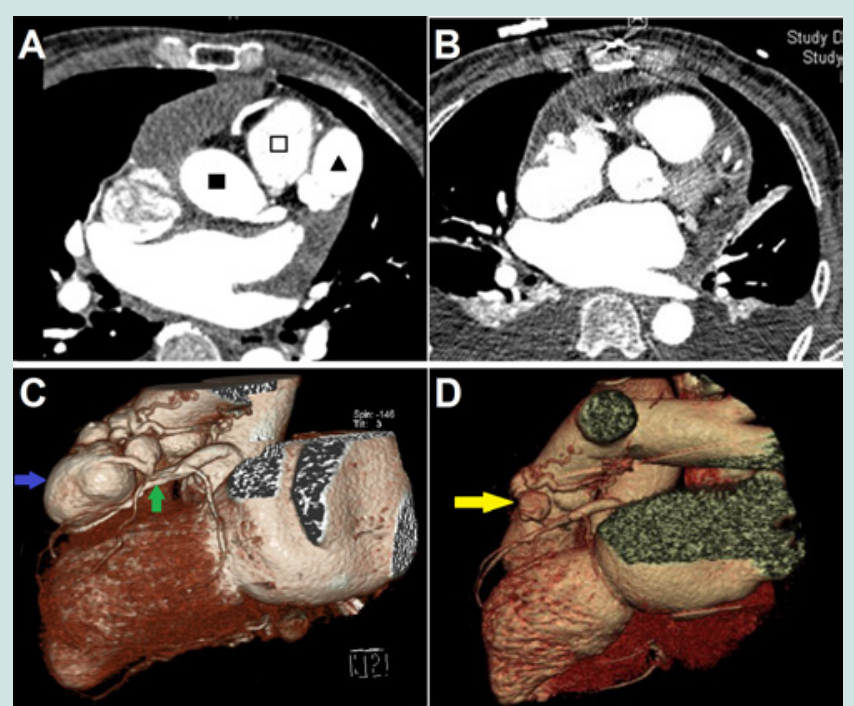

Figure 2: (A) filled triangle indicating a large vascular mass, open square indicating pulmonary artery, and filled square indicating aorta. (B) Changes after the resection of the large vascular mass (a giant coronary artery aneurysm). (C) Blue arrow showing a giant aneurysm $(43 \mathrm{~mm} \times 37 \mathrm{~mm})$ of proximal left anterior descending artery with multiple small aneurysms around it; green arrow indicating the left anterior descending artery. (D) Yellow arrow shows that the giant aneurysm disappeared after surgery.

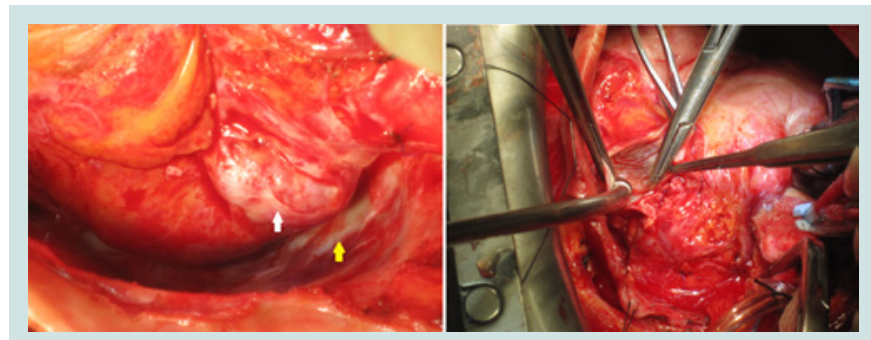

Figure 3: Images of pericardiotomy and resection of the giant coronary artery aneurysm. White arrow indicates the giant coronary artery aneurysm. Yellow arrow shows pericardial thickening.

The operation and histopathological findings confirmed the diagnosis of constrictive pericarditis, which may be caused by tuberculosis and have existed for a period of time. The constrictive pericarditis was less likely to be caused by aneurysm rupture, because CAA rupture commonly leads to acute hemopericardium, cardiac tamponade, and even sudden cardiac death $[9,10]$.

Sick sinus syndrome is characterized by a collection of abnormal heart rhythms including sinus arrest, remarkable sinus bradycardia and sinus node exit block, which are induced by the dysfunction of sinus node. Most of them are chronic process, but in a few cases the disorder of sinus node and cardiac conduction system might be acute occurrence, such as acute myocardial infarction and myocarditis. Under such conditions, the arrhythmias might be reversible, if the treatment is timely. According to this definition and the symptoms in the patient, we considered the possibility of sick sinus syndrome. The sick sinus syndrome in this case was likely to develop within the last two weeks and resulted in exacerbations of symptoms and syncope. Although sick sinus syndrome can be induced by abnormality of congenital artery development [11], congenital artery malformations were less likely to be the cause of sick sinus syndrome in the present case. We have described sick sinus syndrome as a rare complication of constrictive pericarditis and giant CAA rupture. Spodick believed that pericarditis was only associated with significant arrhythmias when there were additional heart diseases or cardiac tamponade [12]. Unexpectedly, the sick sinus syndrome was improved after operation. We raised the hypothesis that the sick sinus syndrome may be caused by giant CAA rupture, constrictive pericarditis and hemopericardium, which induced high pressure in pericardial cavity and compressed the sinus node or its artery. Therefore, the acute sinus dysfunction could be relieved by the cleaning of hemopericardium, pericardiectomy, and the resection of giant aneurysm. However, we could not completely exclude the possibility of functional bradyarrhythmia. Therefore, we will further track this case.

\section{Conclusion}

We reported a rare case of spontaneous rupture of giant CAA in a patient with undiagnosed constrictive pericarditis, with a rare complication of sick sinus syndrome. The case report underlines the significance of multiple non-invasive imaging tests to the diagnosis of CAA and constrictive pericarditis. Emergency surgical intervention is recommended for patients having giant CAA with obvious symptoms or complications like rupture.

\section{References}


Citation: Liu Y, Hua P, Chen J, Zhang HJ, Sun WF, et al. Sick Sinus Syndrome Induced by Giant Coronary Artery Aneurysm Rupture in a Patient with Constrictive Pericarditis. J Cardiobiol. 2014;2(1): 3

1. Halapas A, Lausberg H, Gehrig T, Friedrich I, Hauptmann KE (2013) Giant right coronary artery aneurysm in an adult male patient with non-ST myocardial infarction. Hellenic J Cardiol 54: 69-76.

2. Pahlavan PS, Niroomand F (2006) Coronary artery aneurysm: a review. Clin Cardiol 29: 439-443.

3. Baruteau AE, Martins RP, Boulmier D, Basquin A, Briard D, et al. (2012) Acquired left ventricular submitral aneurysms in the course of Takayasu arteritis in a child. Congenit Heart Dis 7: 76-79.

4. Palmer P (1982) Fatal ruptured mycotic coronary artery aneurysm. P N G Med J 25: 281-282.

5. Albat B, Missov E, Leclercq F, Grolleau R, Thevenet A (1994) Adult coronary aneurysms related to Kawasaki disease. J Cardiovasc Surg (Torino) 35: 57 60.

6. Brevard SB, Smith VC (1990) Presumed Kawasaki disease resulting in multiple coronary artery aneurysms in an adult. Ann Thorac Surg 50: 291-
7. Atik E (2012) Case 1/2012: 55-year-old male with multiple coronary artery aneurysms due to Kawasaki disease. Arq Bras Cardiol 98: e1-2.

8. Li D, Wu Q, Sun L, Song Y, Wang W, et al. (2005) Surgical treatment of giant coronary artery aneurysm. J Thorac Cardiovasc Surg 130: 817-821.

9. Matsuno Y, Fukumoto Y, Ishida N, Shimabukuro K, Takemura H (2013) Mycotic left main coronary artery aneurysm following double-valve replacement for active infective endocarditis. Ann Thorac Cardiovasc Surg 19: $70-72$.

10. Koike R, Oku T, Satoh H, Sawada Y, Suma H, et al. (1990) Right ventricular myocardial infarction and late cardiac tamponade due to right coronary artery aneurysm--a case report. Jpn J Surg 20: 463-467.

11. Wang WB, Young MS, Ding YA (1992) Internal mammary artery to pulmonary artery fistula associated with sick sinus syndrome: report of a case. J Formos Med Assoc 91: 635-638.

12. Spodick DH (1976) Arrhythmias during acute pericarditis. A prospective study of 100 consecutive cases. JAMA 235: 39-41.

\section{Acknowledgements}

This work was supported in part by NSFC [81170647 and 81370837 to Hui Huang, and 81270212 to Jingfeng Wang] and Fok Ying-Tong Education Foundation for Young Teachers in the Higher Education Institutions of China [132030], Yat-sen Scholarship for Young Scientists, the Science \& technology star of Zhujiang (Guangzhou) [2011016] to Hui Huang. 